\title{
On the passage of the wave along the rod
}

\author{
Anastasia Karakozova ${ }^{1,2}$ \\ ${ }^{1}$ Moscow State University of Civil Engineering, Yaroslavskoe shosse, 26, Moscow, 129337, Russia \\ ${ }^{2}$ IPMech RAS, 119526 Vernadskogo prosp. 101/1, Moscow, Russia
}

\begin{abstract}
The article is devoted to the problem of wave damping in elastic media. This study can be of great practical application for use in systems for damping vibration effects, including those caused by shock waves, as well as for damping vibrations caused by seismic wave effects for structures located in seismically hazardous regions as part of seismic protection measures for objects.
\end{abstract}

\section{Introduction}

There is quite a large number of works on static [ $1-6]$ and dynamic [7 - 10] problems of periodic media. Most of them are concerned with homogenization procedures, allowing substitution of a periodic medium by the equivalent homogeneous one. However, dispersion analysis of surface acoustic waves in layered structures reveals substantial discrepancy of dispersion curves in layered and homogenized media [11 - 16]. Herein, it is observed that propagation of the $1 \mathrm{D}$-waves in an elastic rod having stepwise periodic structure with acoustically contrast components, differs considerably from what is expected in an elastic homogeneous rod.

\subsection{Wave theory}

Let's select in the medium a cylindrical volume with a height $\Delta \mathrm{x}$ with a base area $\mathrm{S}$ [16].

Let a longitudinal plane wave propagate in the direction of the $\mathrm{x}$ axis. (Fig. 1). 


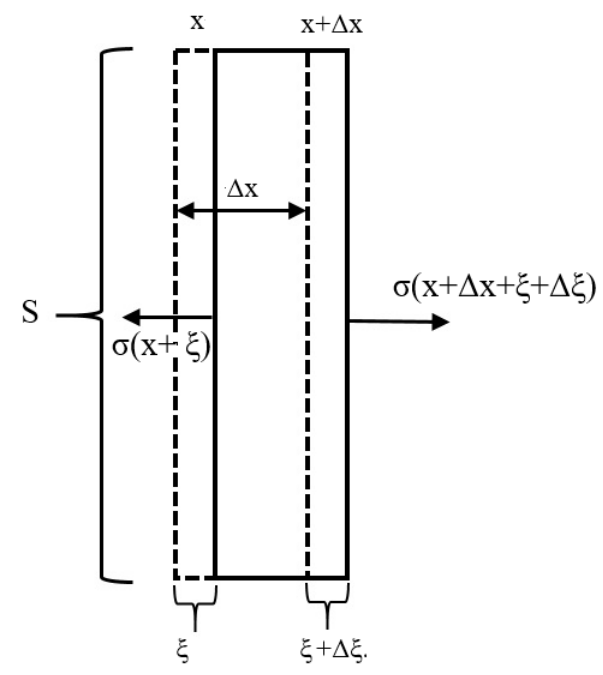

Fig. 1. Wave propagation

Displacements $\xi$ of particles with different $\mathrm{x}$ at each moment of time are different (Fig.2).

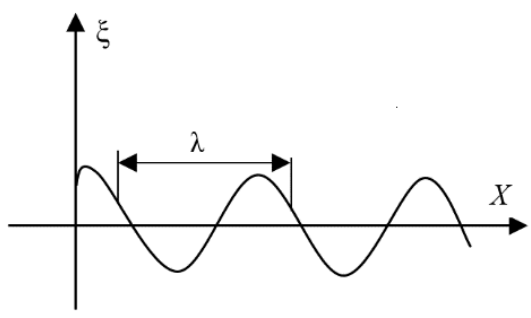

Fig. 2. Displacement $\xi$ of particles from an equilibrium position as a function of distance $X$ measured along the direction of wave propagation, where $\lambda$ is a wavelength.

If the base of the cylinder with the $\mathrm{x}$ coordinate has a displacement $\xi$ at some point in time, then the base displacement with the $\mathrm{x}+\Delta \mathrm{x}$ coordinate will be $\xi+\Delta \xi$. Consequently, the considered volume is deformed - it gets elongation $\Delta \xi(\Delta \xi$-algebraic value; $\Delta \xi<0$ corresponds to compression of the cylinder) or relative elongation $\Delta \xi / \Delta \mathrm{x}$. The value $\Delta \xi /$ $\Delta \mathrm{x}$ gives the average deformation of the cylinder. Due to the fact that $\xi$ changes with a change in $\mathrm{x}$ not according to a linear law, the true deformation in different sections of the cylinder will not be the same. To obtain a deformation in the section $\mathrm{x}$, it is necessary to tend $\Delta \mathrm{x}$ to zero. Hence,

$$
\varepsilon=\frac{d \xi}{d x}
$$

The presence of tensile deformation indicates the existence of normal stress $\sigma$ at small deformations proportional to the deformation value. From the definition of voltage 


$$
\sigma=E e=E \frac{d \xi}{d x}
$$

where $\mathrm{E}$ - is an elastic modulus of the media.

Note that the relative deformation $\frac{d \xi}{d x}$ and, consequently, the stress $\sigma$ at a fixed time depends on $\mathrm{x}$ (Fig.3).

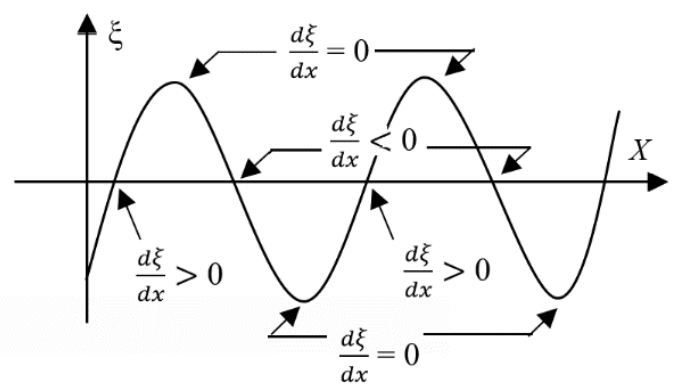

Fig. 3. The graph of $\xi$ on $x$ dependence

Where the deviations of particles from the equilibrium position are maximum, deformation and stress are equal to zero. In places where the particles pass through the equilibrium position, deformation and stress reach their maximum value, with positive and negative deformations (i.e. tension and compression) alternating with each other. In accordance with this, the longitudinal wave consists of alternating rarefaction and thickening of the medium.

Let us turn again to the cylindrical volume shown in Fig. 1, and write the equation of motion for it. Taking $\Delta \mathrm{x}$ very small, the acceleration of the cylinder can be taken equal to $\frac{d^{2} \xi}{d t^{2}}$. The mass of the cylinder is $\rho S \Delta x$, where $\rho$ is the density of the undeformed medium. The force acting on the cylinder is equal to the product of the area of the base of the cylinder $\mathrm{S}$ by the difference between the normal stresses in the section $(x+\Delta x+\xi+$ $\Delta \xi)$ and $(x+\xi)$

$$
f=S E\left[\left(\frac{d \xi}{d x}\right)_{x+\Delta x+\xi+\Delta \xi}-\left(\frac{d \xi}{d x}\right)_{x+\xi}\right]
$$

The value $\left(\frac{d \xi}{d x}\right)_{x+\delta}$ for small $\delta$ can be represented with a high degree of accuracy in the form

$$
\left(\frac{d \xi}{d x}\right)_{x+\delta}=\left(\frac{d \xi}{d x}\right)_{x}+\left[\frac{d}{d x}\left(\frac{d \xi}{d x}\right)\right]_{x} \delta=\left(\frac{d \xi}{d x}\right)_{x}+\frac{d^{2} \xi}{d x^{2}} \delta
$$

where $\frac{d^{2} \xi}{d x^{2}}$ is the value of the second derivative $\xi$ with respect to $x$ in the section $x$.

Due to the smallness of the values $\Delta x, \xi, \Delta \xi$ and we apply transformation (4) to expression (3): 


$$
\begin{gathered}
f=S E\left\{\left[\left(\frac{d \xi}{d x}\right)_{x}+\frac{d^{2} \xi}{d x^{2}}(\Delta x+\xi+\Delta \xi)\right]-\left[\left(\frac{d \xi}{d x}\right)_{x}+\frac{d^{2} \xi}{d x^{2}} \xi\right]\right\}= \\
=S E \frac{d^{2} \xi}{d x^{2}}(\Delta x+\Delta \xi) \approx S E \frac{d^{2} \xi}{d x^{2}} \Delta x
\end{gathered}
$$

(the relative elongation $\frac{d \xi}{d x}$ under elastic deformations is much less than unity. Therefore, $\Delta \xi \ll \Delta x$ the summand $\Delta \xi$ in the sum $(\Delta x+\Delta \xi)$ can be neglected $)$.

Substituting mass, acceleration and force into the equation of Newton's second law, we get:

$$
\rho S \Delta x \frac{d^{2} \xi}{d t^{2}}=S E \frac{d^{2} \xi}{d x^{2}} \Delta x
$$

Finally, canceling by $S \Delta x$, we arrive at the equation

$$
\frac{d^{2} \xi}{d x^{2}}=\frac{\rho}{E} \frac{d^{2} \xi}{d t^{2}}
$$

which is the wave equation

$$
\frac{d^{2} \xi}{d x^{2}}+\frac{d^{2} \xi}{d y^{2}}+\frac{d^{2} \xi}{d z^{2}}=\frac{1}{v^{2}} \frac{d^{2} \xi}{d t^{2}}
$$

written for a particular case when $\xi$ does not depend on $y$ and $z$. Comparing (7) with (8), we find that

$$
v=\sqrt{\frac{E}{\rho}}
$$

Thus, the phase velocity of longitudinal elastic waves $v$ is equal to the square root of Young's modulus divided by the density of the medium.

\section{Numerical experiment}

For the numerical experiment, the Abaqus 6.14 software package was used. To simulate an elastic medium, a cylindrical rod $50 \mathrm{~m}$ long and $0.5 \mathrm{~m}$ wide is considered. It is divided into 20 sections with different elastic properties (Fig.4).

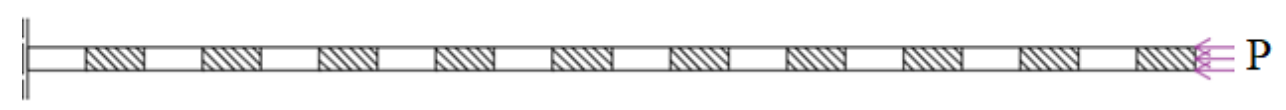

Fig. 4. Bar model schematic with segments

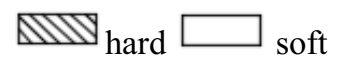

Hard section has density $\rho=100$ units, elastic modulus E $=100 \mathrm{MPa}$ and Poisson's ratio $\mu=0,25$. Soft section has density $\rho=0,01$ units, elastic modulus $E=0,01 \mathrm{MPa}$ and Poisson's ratio $\mu=0,25$. 
The rod is hinged at one of the ends (left end on the Fig.4). The other end of the rod is loaded with a uniform pressure $\mathrm{P}$ that changes over time, and the change over time follows a triangular law (Table 1)

Table 1. A triangular law of the force changing

\begin{tabular}{|c|c|}
\hline $\mathrm{T}, \mathrm{c}$ & $\mathrm{P}, \mathrm{\kappa N}$ \\
\hline 0 & 0 \\
\hline 0,25 & 1 \\
\hline 0,5 & 0 \\
\hline
\end{tabular}

The time of passage of the load along the rod was chosen equal to $50 \mathrm{sec}$, since according to the formula for the rod velocity (10). When substituting the values of the characteristics of the media under consideration, a rod velocity $\mathrm{V}$ was obtained, equal to 1 $\mathrm{m} / \mathrm{s}$, and for a rod $50 \mathrm{~m}$ long, a time of $50 \mathrm{sec}$ was obtained.

Based on the calculation results, graphs of accelerations, displacements and velocities were obtained at points on the boundaries of alternating media (hard / soft).

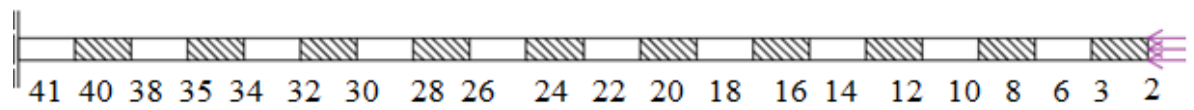

Fig. 5. Numbering of the point in question

\section{Results}

The results of the calculation are shown in the following figures (Fig.6 - Fig.8): graphs of the dependence of acceleration, displacement and velociry from time in logarithmic coordinates.

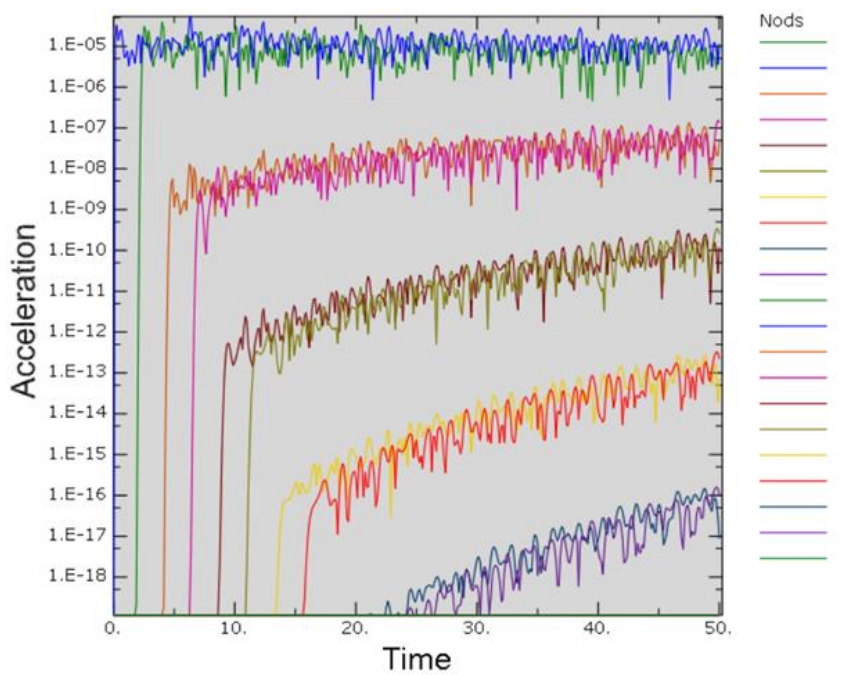

A_ANTIALIASING:Magnitude PI: PART-1-1 N:2_1 A ANTIALIASING: Magnitude PI: PART-1-1 N: 3 A_ANTIALIASING:Magnitude PI: PART-1-1 N: 6 A_ANTLALIASING: Magnitude PI: PART-1-1 N: 8 A ANTIALIASING:Magnitude PI: PART-1-1 N: 10 A_ANTIALIASING: Magnitude PI: PART-1-1 N: 12 A_ANTIALIASING:Magnitude PI: PART-1-1 N: 14 A_ANTIALIASING:Magnitude PI: PART-1-1 N: 16 A_ANTIALIASING: Magnitude PI: PART-1-1 N: 18 A_ANTIALIASING: Magnitude PI: PART-1-1 N: 20 A_ANTIALIASING:Magnitude PI: PART-1-1 N: 22 A_ANTIALIASING: Magnitude PI: PART-1-1 N: 24 A_ANTIALIASING:Magnitude PI: PART-1-1 N: 26 A_ANTLALIASING:Magnitude PI: PART-1-1 N: 28 A_ANTIALIASING: Magnitude PI: PART-1-1 N: 30 A_ANTIALIASING:Magnitude PI: PART-1-1 N: 32 A_ANTLALIASING: Magnitude PI: PART-1-1 N: 34 A_ANTIALIASING:Magnitude PI: PART-1-1 N: 35 A_ANTIALIASING:Magnitude PI: PART-1-1 N: 38 A_ANTIALIASING:Magnitude PI: PART-1-1 N: 40 A_ANTIALIASING:Magnitude PI: PART-1-1 N: 41

Fig. 6. Graph of acceleration (in logarithmic coordinates) versus time 


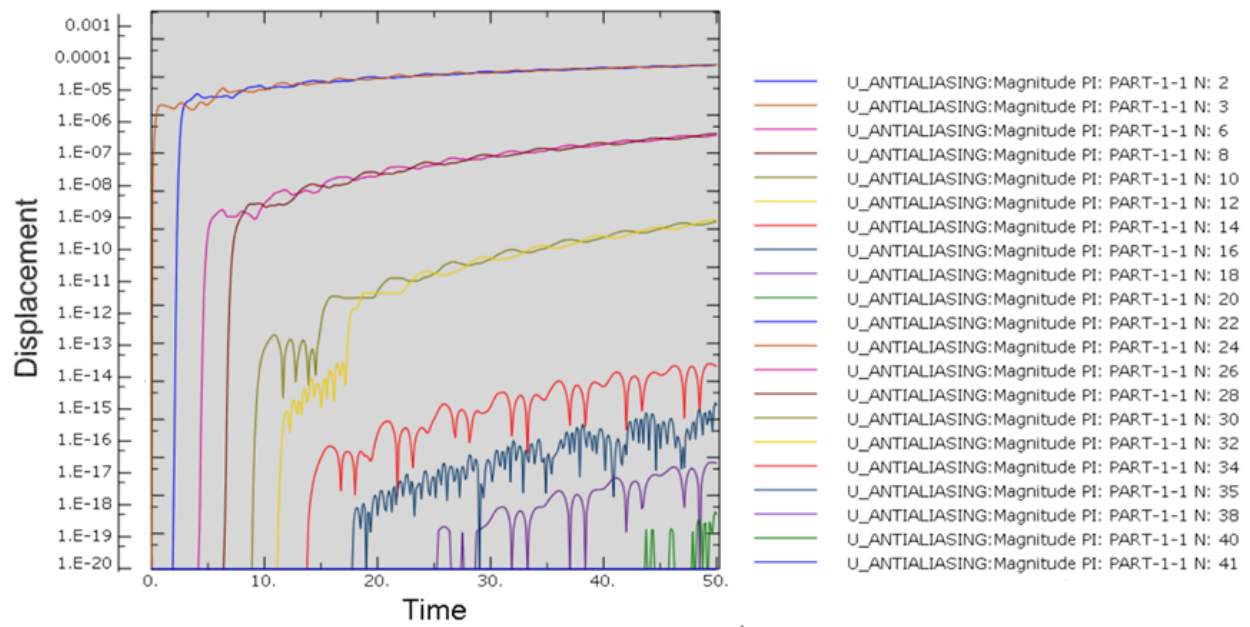

Fig. 7. Graph of the dependence of displacement (in logarithmic coordinates) from time

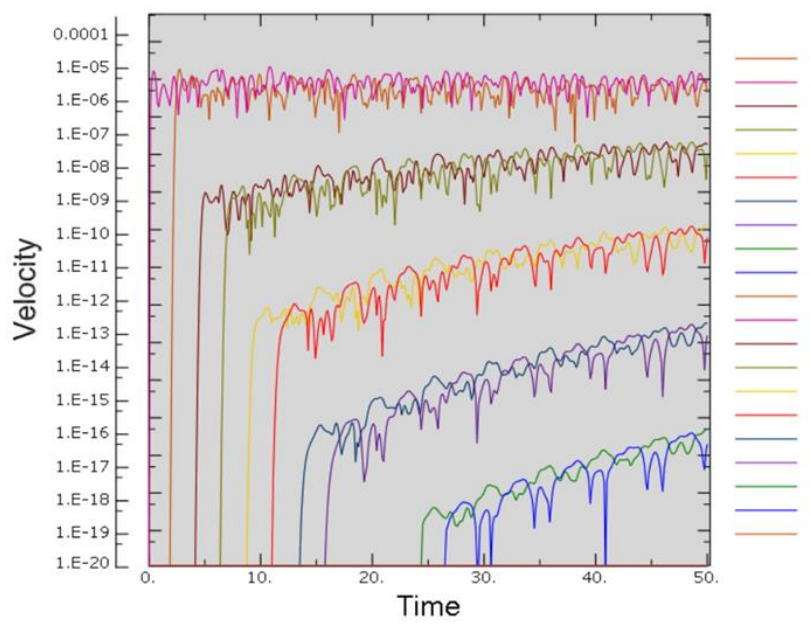
V_ANTIALIASING:Magnitude PI: PART-1-1 N: 2
V_ANTIALIASING:Magnitude PI: PART-1-1 N: 3
V_ANTIALIASING:Magnitude PI: PART-1-1 N: 6
V_ANTIALIASING:Magnitude PI: PART-1-1 N: 8
V_ANTIALIASING:Magnitude PI: PART-1-1 N: 10
V_ANTIALIASING:Magnitude PI: PART-1-1 N: 12
V_ANTIALIASING:Magnitude PI: PART-1-1 N: 14
V_ANTIALIASING:Magnitude PI: PART-1-1 N: 16
V_ANTIALIASING:Magnitude PI: PART-1-1 N: 18
V_ANTIALIASING:Magnitude PI: PART-1-1 N: 20
V_ANTIALIASING:Magnitude PI: PART-1-1 N: 22
V_ANTIALIASING:Magnitude PI: PART-1-1 N: 24
V_ANTIALIASING:Magnitude PI: PART-1-1 N: 26
V_ANTIALIASING:Magnitude PI: PART-1-1 N: 28
V_ANTIALIASING:Magnitude PI: PART-1-1 N: 30
V_ANTIALIASING:Magnitude PI: PART-1-1 N: 32
V_ANTIALIASING:Magnitude PI: PART-1-1 N: 34
V_ANTIALIASING:Magnitude PI: PART-1-1 N: 35
V_ANTIALIASING:Magnitude PI: PART-1-1 N: 38
V_ANTIALIASING:Magnitude PI: PART-1-1 N: 40
V_ANTIALIASING:Magnitude PI: PART-1-1 N: 41

Fig 8. Graph of the dependence of velocity (in logarithmic coordinates) from time

\section{Discussion}

The values under consideration (acceleration $\mathrm{A}$, velocity $\mathrm{V}$ and displacement $\mathrm{U}$ ) have a maximum at point 2 (the point as close as possible to the place of load application). With distance from the place of load application, the distribution graphs of the quantities under consideration tend to zero. At the point closest to the opposite end of the rod (where there is a hinge), the graphs of the values under consideration practically merge with the horizontal axis, that is, they are as close to zero as possible 


\section{Conclusions}

Such an arrangement of areas with alternating elastic properties (Fig.4) contributes to damping vibrations caused by a given load in an elastic medium.

\section{Acknowledgement}

The work was supported by the Russian Science Foundation Grant 20-49-08002.

\section{References}

1. G. Allaire. Homogenization and two-scale convergence. SIAM J. on Mathematical Analysis, 23(6):1482-1518 (1992)

2. M. Bardi and E. Feleqi. Nonlinear elliptic systems and mean-field games. Nonlinear Differential Equations Appl., 23(4):Art. 44 (2016)

3. L. Bufford and I. Fonseca. A note on tw.o scale compactness. Port. Math., 72(23):101-117 (2015)

4. L. Carbone and R. D. Arcangelis. Unbounded functionals in the calculus of variations. Vol. 125. Chapman \& Hall/CRC Monographs and Surveys in Pure and Applied Mathematics. Chapman \& Hall/CRC,Boca Raton, FL, Representation, relaxation, and homogenization (2002)

5. P. Cardaliaguet, J.-M. Lasry, P.-L. Lions, and A. Porretta. Long time average of mean field games. Netw. Heterog. Media, 7(2):279-301 (2012)

6. P. Cardaliaguet, J-M. Lasry, P-L. Lions, and A. Porretta. Long time average of mean field games with anonlocal coupling. SIAM J. on Control and Optimization, 51(5):3558-3591 (2013)

7. J. Aboudi, M.-J. Pindera, and S. Arnold. Linear thermoelastic higher-order theory for periodic multiphase materials. J. of Applied Mech., 68(5):697-707 (2001)

8. A. Bensoussan, J.-L. Lions, and G. Papanicolaou. Asymptotic analysis for periodic structures. North-Holland, Amsterdam (1978)

9. D. Addessi, M. L. De Bellis, and E. Sacco. Micromechanical analysis of heterogeneous materials subjected to overallcosserat strains. Mechanics Research Communications, 54:27-34 (2013)

10. G. Allaire. Homogenization and two-scale convergence. SIAM Journal of Mathematical Analisys, 23:1482-1518 (1992)

11. S. Kuznetsov. SH-waves in laminated plates. Quart. Appl. Math. 64: 153-165, (2006)

12. S. Kuznetsov. Love waves in stratified monoclinic media. Quart. Appl. Math. 62: 749-766 (2004)

13. A. Ilyashenko et al. SH waves in anisotropic (monoclinic) media. Z. Angew. Math. Phys. 69(17) (2018)

14. R.V. Goldstein et al. Long-Wave Asymptotics of Lamb Waves. Mech. Solids 52: 700-707 (2017) 
15. S. Kuznetsov, A. Karakozova. Stress and displacement intensity factors of cracks in anisotropic media. Int. J. of Applied Mechanics and Engineering, vol.25, No.3, 212-218 (2020)

16. S. Kuznetsov. Abnormal dispersion of Lamb waves in stratified media. Z. Angew. Math. Phys. 70(175) (2019)

17. I. V. Saveliev. Course of general physics. Volume I: mechanics, oscillations and waves, molecular physics Moscow (1970) 